\title{
Precursor-induced conditional random fields: connecting separate entities by induction for improved clinical named entity recognition
}

Wangjin Lee ${ }^{1}$ and Jinwook Choi ${ }^{1,2,3^{*}}$ (D)

\begin{abstract}
Background: This paper presents a conditional random fields (CRF) method that enables the capture of specific high-order label transition factors to improve clinical named entity recognition performance. Consecutive clinical entities in a sentence are usually separated from each other, and the textual descriptions in clinical narrative documents frequently indicate causal or posterior relationships that can be used to facilitate clinical named entity recognition. However, the CRF that is generally used for named entity recognition is a first-order model that constrains label transition dependency of adjoining labels under the Markov assumption.

Methods: Based on the first-order structure, our proposed model utilizes non-entity tokens between separated entities as an information transmission medium by applying a label induction method. The model is referred to as precursor-induced CRF because its non-entity state memorizes precursor entity information, and the model's structure allows the precursor entity information to propagate forward through the label sequence.

Results: We compared the proposed model with both first- and second-order CRFs in terms of their $\mathrm{F}_{1}$-scores, using two clinical named entity recognition corpora (the i2b2 2012 challenge and the Seoul National University Hospital electronic health record). The proposed model demonstrated better entity recognition performance than both the first- and second-order CRFs and was also more efficient than the higher-order model.

Conclusion: The proposed precursor-induced CRF which uses non-entity labels as label transition information improves entity recognition $F_{1}$ score by exploiting long-distance transition factors without exponentially increasing the computational time. In contrast, a conventional second-order CRF model that uses longer distance transition factors showed even worse results than the first-order model and required the longest computation time. Thus, the proposed model could offer a considerable performance improvement over current clinical named entity recognition methods based on the CRF models.
\end{abstract}

Keywords: Clinical named entity recognition, Conditional random fields, High-order dependency, Clinical natural language processing, Induction method

\footnotetext{
* Correspondence: jinchoi@snu.ac.kr

1 Interdisciplinary Program for Bioengineering, Graduate School, Seoul

National University, 103 Daehak-ro, Jongno-gu, Seoul 03080, South Korea

${ }^{2}$ Department of Biomedical Engineering, Seoul National University College of

Medicine, 103 Daehak-ro, Jongno-gu, Seoul 03080, South Korea

Full list of author information is available at the end of the article
}

(c) The Author(s). 2019 Open Access This article is distributed under the terms of the Creative Commons Attribution 4.0 International License (http://creativecommons.org/licenses/by/4.0/), which permits unrestricted use, distribution, and reproduction in any medium, provided you give appropriate credit to the original author(s) and the source, provide a link to the Creative Commons license, and indicate if changes were made. The Creative Commons Public Domain Dedication waiver (http://creativecommons.org/publicdomain/zero/1.0/) applies to the data made available in this article, unless otherwise stated. 


\section{Background}

With the recent application of artificial intelligence to the medical field, health information systems are expected to handle medical data in the form of unstructured text. The unstructured clinical text conveys descriptions of patients' health information, including their histories of illness and hospital treatment. Salient concepts that express a patient's health status are represented by named entities (NEs) in the text. The identifying textual mentions of health-related concepts, termed clinical named entity recognition (NER), is a sub-problem in the field of clinical natural language processing (NLP) [1]. The health information that requires identification can range from a single entity to an elaborate description containing many entities. Heterogeneous classes of clinical entities have been employed in recent studies; these are strongly related to clinical activities, such as medical examination, medication, and diagnosis [2-7].

The NER problem consists of identifying spans of entities and attaching labels indicating the appropriate semantic class, as shown in Fig. 1. In the NER task, the text can be seen as a word (or token) sequence, and the most advanced NER models are therefore based on sequence labeling approaches that use machine learning methods $[3,4,8,9]$. The concept of conditional random fields (CRFs) [10] has demonstrated promising results in many sequence labeling problems, including NER [3,10-14], as well as a deep learning architecture applied to the NER task $[15,16]$. CRF models are particularly effective for text processing because they learn transition factors between labels of single tokens, assuming that the current label is conditioned on both current observations and the immediately preceding label. The first-order constraint is applied in order to reduce computational complexity and to maintain the model's simplicity.

However, the constraint on the labels' adjacency prevents the model from expressing transition dependency between entities separated by a long distance. NEs tend to be separated by non-entity words in NER problems, and this innate attribute inhibits the first-order CRF model's ability to capture dependencies between NE labels when the two entities are separated by non-entities that are outside tokens [17]. Therefore, CRF models using the first-order transition factor have difficulty in capturing higher-order interdependencies of NEs.

More specifically, we assumed that 1) named entities are prevalent in clinical texts, 2) the entities in clinical texts are semantically related, thus the information of preceding entity's label would be an important feature for an NER model's prediction of a certain label (at a time step), 3) the labeling mechanism of the CRF model that uses label transition information as one important feature would be suitable for clinical NER. However, according to the study published by Dan Roth's group (CoNLL 2009), it is limited to use the transition information, especially for NER in the first- or second-order model based on Markov assumption because the named entities are generally separated each other in a text.

Previous NER studies have focused on methods of exploring long-distance dependencies in NER while maintaining computational tractability. Conventional highorder CRFs is known to be intractable in practice because they multiply the feature space and require more training data to prevent the data sparseness problem [18]. Sarawagi and Cohen proposed a semi-Markov CRF [19] that treated the same consecutive labels as a segment and used the label transition between adjoining segments. Subsequent studies have proposed using pre-defined label patterns to implement high-order CRFs [20-22]. However, these methods suffer from limitations associated with the management of entity transitions within non-entity labels of arbitrary length.

This study focuses on using the interdependency of NEs separated by an arbitrary number of non-entity tokens, a condition that is predominant in clinical texts but rarely captured by first-order CRF models. In order to minimize the increase in the model's computational complexity associated with the extraction of longdistance label transition information, this study proposes

Original text (punctuation is separated)

2 years ago NR OPD due to mixed dementia F/U donepezil 1t qd medication .

3 months ago ER visit due to sudden weakness, he has been taken aspirin+Plavix medication .

Perspective of tokens and semantic labels

\begin{tabular}{|c|c|c|c|c|c|c|c|c|c|c|c|c|c|c|c|}
\hline token & 2 & years & ago & NR & OPD & due & to & mixed & dementia & $\mathrm{F} / \mathrm{U}$ & donepezil & $1 \mathrm{t}$ & qd & medication & . \\
\hline label & \multicolumn{3}{|c|}{ Tiime } & \multicolumn{2}{|c|}{ VIsit } & $O$ & $O$ & \multicolumn{3}{|c|}{ Disease } & \multicolumn{4}{|c|}{ Drug } & $O$ \\
\hline
\end{tabular}

\begin{tabular}{|c|c|c|c|c|c|c|c|c|c|c|c|c|c|c|c|c|c|}
\hline token & 3 & months & ago & ER & visit & due & to & sudden & weakness & , & he & has & been & taken & Aspirin + Plavix & medication & \\
\hline label & & Time & & Visit & $O$ & $O$ & $O$ & & uptom & $O$ & $O$ & $O$ & $O$ & $O$ & Drug & & $O$ \\
\hline
\end{tabular}

Fig. 1 NER perspective of a text; the label $O$ represents a non-entity 
an induction method that allows information to propagate from one state to state between two entities through non-entity sequence within a single instance.

Concentrating on the CRF model study rather than the medical NER, this paper would briefly introduce recent studies in medical NER. Deep-learning based methods for clinical concept identification are actively studied especially based on recurrent neural network structures [16, 23-28]. In the long short-term memory and CRF architecture, the CRF is still used for labeling of a sequence because the CRF model can jointly use neighboring tags in its output decision [15]. In order to automate medical NER a research [29] has been proposed to incorporate active learning. Once named entities are extracted, the identified terms can be utilized in order to derive more information beyond textual data, such as temporal information extraction [3, 30], drugdisease relationship recognition from large scale medical literature [31], and identification of risk factors related to a particular disease [32]. In order to support researchers requiring NER modules, off-the-shelf medical NER programs are recently published such as CLAMP [33] and MetaMap Lite [34].

The remainder of this paper is organized as follows. The Methods section details the proposed CRF model and the model evaluation method. The Results section presents the evaluation results, and the Discussion section considers several observations related to the use of the proposed model in clinical NER. The Conclusion section summarizes the study's main findings.

\section{Methods}

\section{Conditional random fields}

In the conventional CRF model applied to NER, a textual instance (i.e., sentence) can be represented as a pair $(\boldsymbol{x}, \boldsymbol{y})$ where $\boldsymbol{x}$ is an observed feature sequence including one or more words (tokens) and $\boldsymbol{y}$ is the feature sequence's corresponding label sequence. Because the text is a linear sequence of tokens, the CRF for NER takes the form of a linear chain. The length of $x$ is the number of tokens, and the sequence $\boldsymbol{y}$ has the same length as $\boldsymbol{x}$. The label is hidden, and a hidden state value set consists of the target entity labels and a single non-entity label for non-entity tokens. The CRF model then represents the conditional distribution $P(\boldsymbol{y} \mid \boldsymbol{x})$ as an equation of feature functions as follows:

$$
\mathrm{p}(y \mid x)=\frac{1}{Z(x)} \cdot \prod_{t=1}^{T} \exp \left\{\sum_{k=1}^{K} \theta_{k} f_{k}\left(y_{t}, y_{t-1}, x_{t}\right)\right\}
$$

where $f_{k}$ is a $k^{\text {th }}$ arbitrary feature function having the corresponding weight $\theta_{k}, K$ is the number of feature functions, $t$ is the time step, $T$ is the number of tokens in an instance of $x$, and $Z(x)$ is a partition function summing the numerator for all possible $y$ sequences [35]. The learning objective is to find the weight set that maximizes the conditional distribution. The function $f_{k}$ is a binary indicator function that has a value of 1 only if the function matches the target condition, and is otherwise 0 . Dependencies between random variables are presented in the form of feature function $f_{\mathrm{k}}$ in the CRF; the feature functions are either transition factors or observation factor functions. The transition factors in the CRF model take the form of $f_{k}{ }^{\mathrm{ij}}\left(y, y^{\prime}, x\right)=\mathbf{1}_{\{\mathrm{y}=\mathrm{i}\}} \mathbf{1}_{\left\{\mathrm{y}^{\prime}=\mathrm{j}\right\}}$ where $i$ and $j$ are certain label symbols having transition relationship according to this function. The observation factors takes the form as Eq. (2) where $i$ and $o$ are certain symbols having an explicit relationship according to this function:

$$
f_{k}^{\text {io }}\left(y, y^{\prime}, x\right)=\mathbf{1}_{\{\mathrm{y}=\mathrm{i}\}} \mathbf{1}_{\{\mathrm{x}=\mathrm{o}\}} \cdot \cdot
$$

Based on this definition of the feature function, the CRF model explicitly represents not only observation information but also label transition information for sequence labeling. For instance, presume a set $\{A, B, O\}$ as the label symbol set; assign $A$ or $B$ to NEs, assign a label symbol $O$ to non-entity tokens, and presume a label sequence of length $4,[A, B, O, B]$, where the first occurrence of entity $B$ follows entity $A$, and a single non-entity token exists between the two entity $B$ s. The first-order CRF models only those label transitions between adjoining state labels, that is, the label transition data $\{(A, B),(B, O),(O, B)\}$, in which the transition between labels $A$ and $B$ is explicitly expressed. Presume another label sequence $[A, O, \ldots O, B]$ where entity $A$ precedes entity $B$ by some distance and an arbitrary length of consecutive non-entity tokens are between the two NEs. The first-order CRF model learns only the label transitions $\{(A, O),(O, O),(O, B)\}$ from the data, in which the dependency $(A, B)$ is not explicitly captured by the model and the fact that entity $A$ precedes entity $B$ is not learned during the training time. Because the CRF model treats single observation tokens as single time steps in a sequence, the gap size between two separate entities is broadened by the number of intermediary non-entities, as shown in Fig. 2.

In Fig. 2, each circle denotes a random variable for labels, and each edge denotes that there is a dependency between connected random variables. In this structure, labels have dependency only between neighbors. Thus a dependency for entity prediction between the label symbols 'Symptom' and 'Drug' for predicting the word 'ASA' seems to be ignored. In the case of the 'ASA', we suspected that the preceding label information could provide additional information for prediction of a particular label for the word if the information can be delivered forward. 


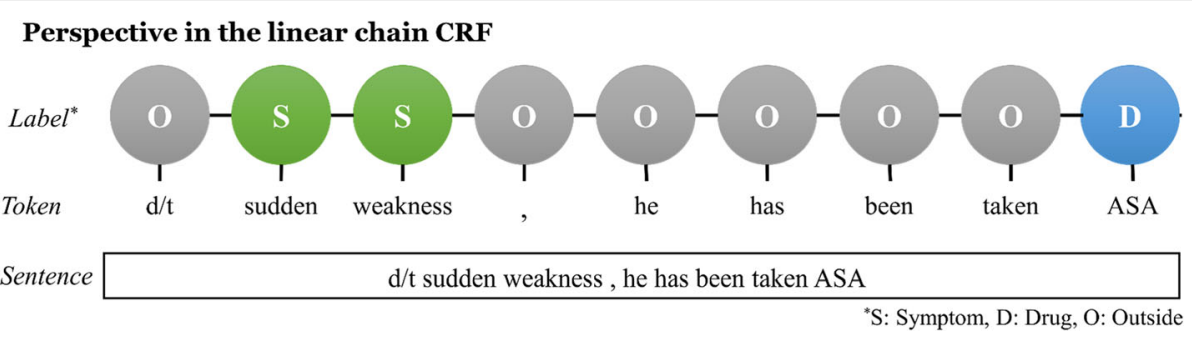

Fig. 2 Example of entities separated by non-entity words in the CRF model (S: symptom; D: drug; O: non-entity)

\section{Precursor-induced conditional random fields}

In order to improve the CRF model for NER applications, this study introduces a precursor-induced CRF (pi-CRF) model to capture specific long-distance transition dependencies between two NEs separated by multiple non-entities. The pi-CRF model:

- Uses non-entity labels to propagate transition information between separated NEs;

- Retains the first-order model structure to reduce the model's computational complexity than the secondorder or higher-order CRF;

- Focuses on label subsequences with the [entity, outside ${ }^{+}$, entity] pattern, as shown in Fig. 3 (a), where the outside $e^{+}$notation denotes one or successive non-entity label symbols;

- Adds a memory element to the hidden state variables to represent those states labeled as nonentities, such that the initial outside label in a non-entity subsequence propagates its explicit first-order dependency on its adjacent entity to the next outside label, which in turn propagates the information to the next outside label, as shown in Fig. 3 (b); and

- Uses an induction process to transmit the information from the first entity through multiple outside label sequence to the second entity state, even though the model uses the first-order dependency (Fig. 3 (b)).

- Modifies the observation feature functions of the CRF in order to share observation symbols among outside label symbols (Eq. 4).

\section{Label induction}

In the pi-CRF, a state with an outside label binds with an additional memory element and behaves as an information transmission medium, delivering information about the presence or absence of the preceding entity forward, which requires the expansion of the hidden state value set (label symbols). The entity label symbols are collected from the training data, and the expanded state value set is eventually derived by a concatenation of

\section{(a) Label sequences of named entities separated by non-entities}

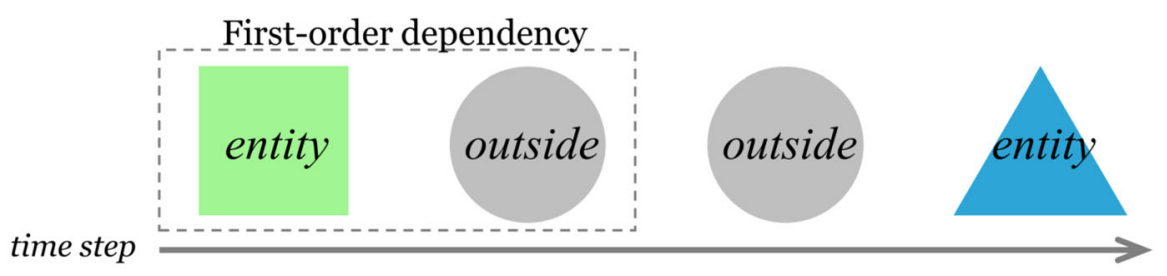

(b) Concept of the propagation of precursor-label information in the pi-CRF

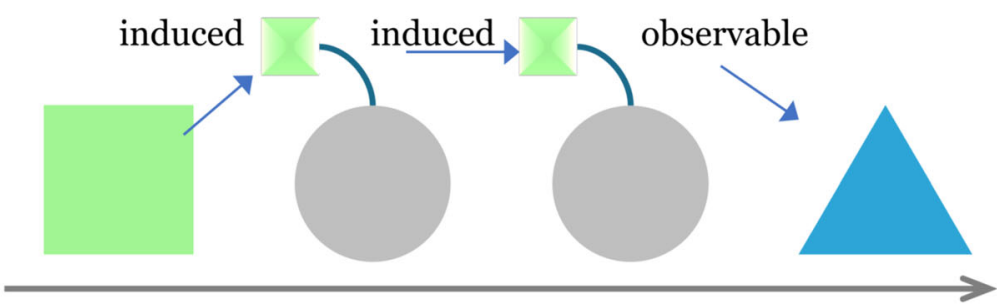

Fig. 3 The transformation from conventional first-order CRF to precursor-induced CRF 
entity label symbol and the outside label symbol. The concatenated outside label symbols thus indicate that the outside label follows a specific entity label. As a naming convention, we use label[OO$]^{+}$to implicitly indicate that the sequence of $O$ (outside) labels follows the concatenated label series. In the example, the symbol $A[\mathrm{O}]^{+}$is one outside label symbol that indicates that an entity $A$ precedes itself, and $O[O]^{+}$is one fragmented outside label symbol indicating that no entity has occurred before this non-entity state. The CRF models distinguish the features for observation symbols and the label symbols. Thus, any types of label symbols do not violate the token symbols, and any label naming convention can be used.

The form of the pi-CRF is derived from Eq. (1), and the conditional probability distribution of the CRF model extension takes the form of feature functions as follows:

$$
\mathrm{p}(y, a \mid x)=\frac{1}{Z(x)} \cdot \prod_{t=1}^{T} \exp \left\{\sum_{k=1}^{K} \theta_{k} f_{k}\left(y_{t}, y_{t-1}, x_{t}, a_{t}, a_{t-1}\right)\right\},
$$

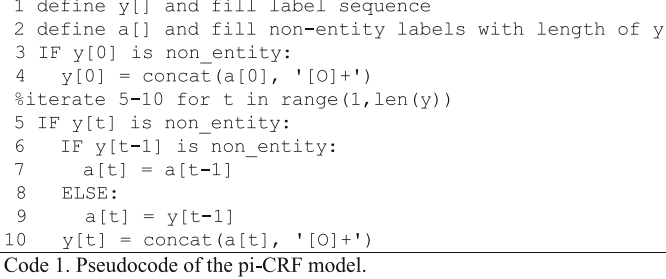

where the variable $a$ stores the induced label information, and the value of $a_{\mathrm{t}}$ is activated by the value of $a_{\mathrm{t}-1}$ and $\mathrm{y}_{\mathrm{t}}$. The conjoined variables $a$ and $y$ are eventually used to derive a newly induced label sequence: once $a_{\mathrm{t}}$ is activated, $a_{\mathrm{t}}$ transmutes the value of $\mathrm{y}_{\mathrm{t}}$ (see the Code 1 ). Based on this model, the dependency of label transition is engaged within only adjacent tokens (i.e., $y_{t}$ and $y_{t-1}$ ) because this model is designated to keep the first-order structure. Thus, the information exists flows forward with the induced outside label by the first-order transition. This structure makes the conveyed information flows forward regardless of the distance.

This induction process subsequently expands the original label symbol set inside the model, producing newly induced and multiple outside label symbols instead of the single outside label symbol. For example, the process modifies an original label sequence $[A, O, \cdots O, B]$ to $[A$, $\left.A[O]^{+}, \cdots A[O]^{+}, B\right]$ according to Code 1 . This transformation helps the model learn long-distance transitions between successive NEs even in the first-order form: from the modified example sequence, the model can learn label transition data $\left\{\left(A[O]^{+}, B\right)\right\}$ where the entity $B$ depends on the non-entity taking entity $A$ as its precursor. This process also generates a trellis structure (Fig. 4 (c)) that is slightly more complex than the trellis generated by the conventional first-order CRF model (Fig. 4 (a)), but simpler than the trellis generated by a conventional second-order CRF model (Fig. 4 (b)). The CRF models generally have as many hidden state options (represented by the nodes in Fig. 4) as there are variables at each time step, and each combination of hidden states denotes a path forward. If $N$ is the number of hidden states in the original first-order CRF model, the pi-CRF model introduces $N$ additional new states; however, this increase in computational complexity is relatively moderate compared to the increase induced by second- or higher-order CRF models. In addition, if the IOB2 tagging scheme [36] is applied to the pi-CRF model, the increase in the number of newly induced hidden states is halved.

One of the main factors determining the CRF model's complexity is the model's graphical structure. The structure can be presented in the form of a tuple. Thus, the structures of the first-order CRF can be presented in (a) Trellis of the first-order CRF

0

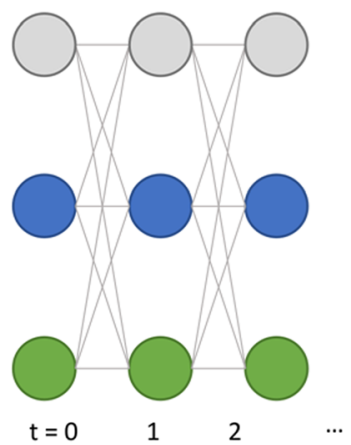

(b) Trellis of the second-order CRF

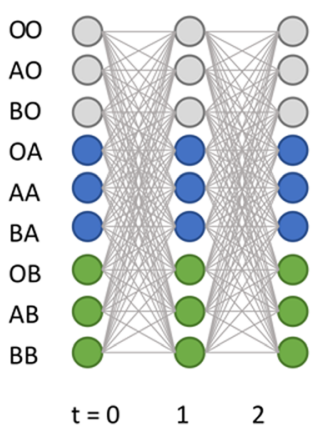

(c) Trellis of the precursor-induced-CRF

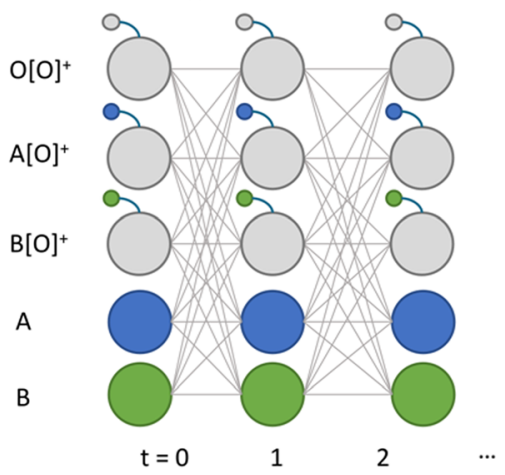

Fig. 4 Trellis graphs generated by different CRFs; each circle indicates random hidden state variables at each time step, and lines indicate the transition paths among the labels. The small circles in (c) are the memory elements added to the hidden states for the non-entity label 
$\left(y_{\mathrm{t}-1}, y_{\mathrm{t}}, x_{\mathrm{t}}\right)$. Because the relationship between $y_{\mathrm{s}}$ is related to transition, the number of transition pair $\left(y_{\mathrm{t}-1}, y_{\mathrm{t}}\right)$ can be $N^{2}$. It means that at least $N^{2}$ calculations are required for each time step of a sequence in both of the training and testing time. In the same way, the graphical structure of the second-order CRF can be presented in $\left(y_{\mathrm{t}-2}, y_{\mathrm{t}-1}, y_{\mathrm{t}}, x_{\mathrm{t}}\right)$ and the transition pair $\left(y_{\mathrm{t}-2}, y_{\mathrm{t}-1}, y_{\mathrm{t}}\right) \mathrm{de}-$ rives at least $N^{4}\left(=N^{2}\right.$ times $\left.N^{2}\right)$ calculations for each time step in training and testing the second-order model. According to the formulation of the pi-CRF (Eq. 2 ), the variable $a$ does not act as a hidden variable but interacts with the variable $y$ in order to expand the possible values of the variable $y$. This system allows the piCRF to operate in the first-order structure and it keeps the model's complexity feasible.

\section{Observation symbol sharing}

It is worth addressing one of the attributes of the piCRF. The model uses modified observation feature functions. The observation feature function $f_{\mathrm{k}}^{\text {io }}$ (Eq. 2) directly implies that a certain label $i$ has 'one-to-one' relationship with a certain observation symbol $o$. If a label symbol does not have a relationship with a particular observation symbol, its relationship is not trained.

The label induction process makes multiple outside label symbols (i.e., 'label[O]+' symbols), instead of using one single outside symbol (i.e., 'O' symbol for the outside label). This induction process would interrupt an outside label symbol to have relationships with whole observation symbols related to non-entities.

Finally, each outside label symbol has relationships with only a portion of observation symbols. For the same training data, it is generally known that machine learning models with more hidden states are more likely to experience data sparseness problems because of their increased feature dimensions [37]. Likewise, in our development period, we observed that the first-order CRF performs worse if the conventional model was trained with the induced label pattern.

In order to prevent the performance decrease, the multiple outside symbols are allowed to share an observation symbol each other in the pi-CRF model, according to the following observation feature function:

$$
f_{k}{ }^{i o}\left(\mathrm{y}, \mathrm{y}^{\prime}, \mathrm{x}\right)=\mathbf{1}_{\{x=o\}} \cdot\left(\mathbf{1}_{\{i \in \text {-outside and } y=i\}}+\mathbf{1}_{\{i \in \text { outside and } y \in \text { outside }\}}\right)
$$

The second and the third indicator terms in the righthand side determine whether the $y$ value is an outside label symbol or not. If the $i$ (the corresponding label symbol of the function $f_{\mathrm{k}}$ ) is not outside symbol, then this equation tests whether the $y$ value is equal to $i$. Contrary, if the $i$ is an outside symbol, then the third indicator term has value 1 as long as the value of the $y$ is an outside symbol. Unlike the feature functions in the conventional CRF constrain 'one-to-one' relationship between a label symbol and an observation symbol in a feature function, the third indicator term allows 'manyto-one' relationship between whole outside label symbols and one observation symbol.

In the pi-CRF, the model used the Eq. (4) for its observation feature function instead of using the Eq. (2) that is used in the conventional CRF. By way of illustration, presume a token, "doctor," occurred with three outside label symbols $\left(\mathrm{O}[\mathrm{O}]^{+}, \mathrm{A}[\mathrm{O}]^{+}\right.$, and $\left.\mathrm{B}[\mathrm{O}]^{+}\right)$in the training set. According to the definition of the observational feature function constraining one-to-one relationship, a first-order CRF has three distinct feature functions $f_{\mathrm{a}}^{\text {io }}\left(x=\right.$ doctor, $\left.y=\mathrm{O}[\mathrm{O}]^{+}\right), f_{\mathrm{b}}^{\text {io }}\left(x=\right.$ doctor, $\left.y=\mathrm{A}[\mathrm{O}]^{+}\right)$, and $f_{\mathrm{c}}^{\text {io }}\left(x=\right.$ doctor, $\left.y=\mathrm{B}[\mathrm{O}]^{+}\right)$. Although the original CRF treats the three feature functions independently, the piCRF has one single feature function for the observation symbol and the outside label symbols, for instance, $f_{\mathrm{k}}^{\text {io }}(x=$ doctor, $y=$ outside symbol $)$.

\section{Model implementation}

Both the original and the pi-CRF models were implemented using Java. The basic CRF structure and algorithms were implemented in MALLET [38]. The pi-CRF model was trained using the original linear chain CRF algorithms without modification because the graphical architecture of the pi-CRF model is fixed as a template for each time step in the same manner as in the original CRF model. In order to train the pi-CRF model, the L-BFGS optimization method [12] and l2-regularization [39] were used to exploit the conventional CRF model's most advantageous features [35]. Furthermore, the Viterbi algorithm was used for inferences from unlabeled sequences. The executable files are available online. ${ }^{1}$

\section{Parameter tuning}

In order to train both models properly, the model parameters were regularized during the development phase. In both the original and the pi-CRF models, 12 regularization [39] was used in order to avoid overfitting, and the form of regularization is as that in Eq. 5:

$$
-\sum_{k=1}^{K} \frac{\theta_{k}^{2}}{2 \sigma^{2}}
$$

where $K$ is the number of feature functions and $\theta_{k}$ is the weight of the $k^{\text {th }}$ feature function $f_{\mathrm{k}}$, and $\sigma$ is the hyper-parameter for the regularization that adjusts the amount of penalty. The regularization term is applied to a log-likelihood form of the CRF models and penalizes large weights.

\footnotetext{
${ }^{1}$ The executable jar files are available in https://github.com/jinsamdol
} 
During the model development process, the training data were split by $8: 2$ for each training and development set and the parameter $\sigma$ was chosen to provide the best $\mathrm{F}_{1}$-score for the development set. The parameter tuning was independently performed on each data set, and the third feature set was used during the tuning process.

\section{Results}

\section{Dataset description}

All the experiments were performed on the NER sets in clinical and general domains: English clinical texts (i2b2 2012 NLP shared task data [3]), rheumatism patients' discharge summaries obtained from Seoul National University Hospital (SNUH) [40], and the CoNLL-2003 NER shared task corpus [41]. The documents in the SNUH set were written using English and Korean. The discharge summaries were annotated using the IOB2 tagging scheme [36].

Although the original annotation in the i2b2 2012 data contains more semantic classes, this evaluation was conducted using the problem, test, and treatment entities. For the SNUH corpus, the entities of symptom, disease, clinical lab test, medication, and procedure/operation were used. We are interested in identifying clinical events related to a patient's clinical events. Thus, we used the clinical semantic classes listed above in our evaluation. For the CoNLL-2003 data, the entities of location, person, organization, and miscellaneous were annotated from the general domain news articles.

Tables 1 and 2 show the data and annotation statistics for each data set. The training and testing sets in the i2b2 2012 and the CoNLL-2003 NER sets were divided following the official distribution set by the data source administrators.

As we assumed that a significant portion of the NEs is separated in sentences, we measured the word distance between the entities in the data sets. The distance dependency was measured within each instance. Table 3 shows examples of the distances between entities in the i2b2 corpus and Fig. 5 shows the distributions of distances between entities in the entire data set for each corpus. The median distance value between entities was 3 and the mean values were within the range of from 3 to 5 , indicating that the NEs in the data sets tended to be separated by 3 to 5 non-entity tokens. The data also indicates that the number of entities within the first-

Table 1 Data specification

\begin{tabular}{lllllll}
\hline Corpus & Domain & Set & Article & Sentence & Token & Entity \\
\hline i2b2 2012 & Clinical & Train & 190 & 7,258 & 94,836 & 11,239 \\
& & Test & 120 & 5,547 & 78,564 & 9,623 \\
\multirow{2}{*}{ SNUH } & \multirow{2}{*}{ Clinical } & Train & 196 & 11,669 & 116,402 & 18,383 \\
& & Test & 193 & 11,042 & 107,666 & 17,125 \\
CoNLL 2003 & \multirow{2}{*}{ General } & Train & 946 & 14,987 & 203,621 & 23,499 \\
& & Test & 231 & 3,684 & 46,435 & 5,629 \\
\hline
\end{tabular}

Table 2 Annotation statistics

\begin{tabular}{|c|c|c|c|c|c|}
\hline \multicolumn{6}{|c|}{ a) $i 2 b 22012$} \\
\hline Set & Problem & \multicolumn{2}{|l|}{ Test } & \multicolumn{2}{|l|}{ Treatment } \\
\hline Train & 4,962 & \multicolumn{2}{|l|}{2,558} & \multicolumn{2}{|l|}{3,719} \\
\hline Test & 4,270 & \multicolumn{2}{|l|}{2,140} & \multicolumn{2}{|l|}{3,213} \\
\hline \multicolumn{6}{|c|}{ b) SNUH } \\
\hline Set & Symptom & Test & Disease & Medication & Procedure \\
\hline Train & 3,923 & 4,559 & 5,084 & 3,642 & 1,175 \\
\hline Test & 3,737 & 3,917 & 4,828 & 3,496 & 1,147 \\
\hline \multicolumn{6}{|c|}{ c) CoNLL 2003} \\
\hline Set & Location & Person & Organization & \multicolumn{2}{|l|}{ Miscellaneous } \\
\hline Train & 7,140 & 6,600 & 6,321 & \multicolumn{2}{|l|}{3,438} \\
\hline Test & 1,656 & 1,617 & 1,662 & \multicolumn{2}{|l|}{694} \\
\hline
\end{tabular}

order range is less than the number of entities within the second- or higher-order ranges. In addition, the ratios of the number of entities having transition dependency to the total number of entities were $0.85,0.73$, and 0.78 for i2b2 2012, SNUH, and CoNLL2003 data sets, respectively. These values indicate that in most cases, entities tend to be interrelated in an instance, rather than being present as single entities.

\section{Feature settings}

Three types of feature settings were investigated in this evaluation, as summarized in Table 4 . The setting \#1 is the simplest available, and the setting \#2 is the configuration in which character-wise prefixes and suffixes could be exploited. Although these two settings use only simple features, these configurations reduce the potential bias that the features could exert on the performance comparison. The setting \#3 implemented features used in previous evaluations of NER methods for each data set [17, 40, 42]; some particular features that are easy to implement were selected for use here. Also, "Token" and "n-gram" are typical features used in NER. The morphologic information used included character-wise affixes (i.e., the first two characters of a token), capitalization patterns (e.g., all

Table 3 Example sentences of the entity distances (single: entity not having a precursor)

\begin{tabular}{|c|c|}
\hline Type & Example sentence with entity annotation \\
\hline single & $\begin{array}{l}\text { The patient is a 28-year-old woman who is } \\
\text { [HIV positive }]_{\text {problem }} \text { for } 2 \text { years. }\end{array}$ \\
\hline $\begin{array}{l}\text { distance } \\
0\end{array}$ & $\begin{array}{l}\text { With [intravenous hydration }]_{\text {treatment }}[\text { the BUN] }]_{\text {test }} \\
\text { and ... }\end{array}$ \\
\hline $\begin{array}{l}\text { distance } \\
1\end{array}$ & $\begin{array}{l}\text {... because of }[\text { pancytopenia }]_{\text {problem }} \text { and } \\
{[\text { vomiting }]_{\text {problem }} \text { on }[\mathrm{DDI}]_{\text {treatment }}}\end{array}$ \\
\hline $\begin{array}{l}\text { distance } \\
8\end{array}$ & $\begin{array}{l}\text { She was brought in for [an esophagogastroduodenoscopy }]_{\text {test }} \\
\text { on } 9 / 26 \text { but she basically was not sufficiently } \\
\text { [sedated] }]_{\text {treatment }} \text { and readmitted at this time for } \\
\text { [a Gl work-up] }]_{\text {test }} \text {. }\end{array}$ \\
\hline
\end{tabular}



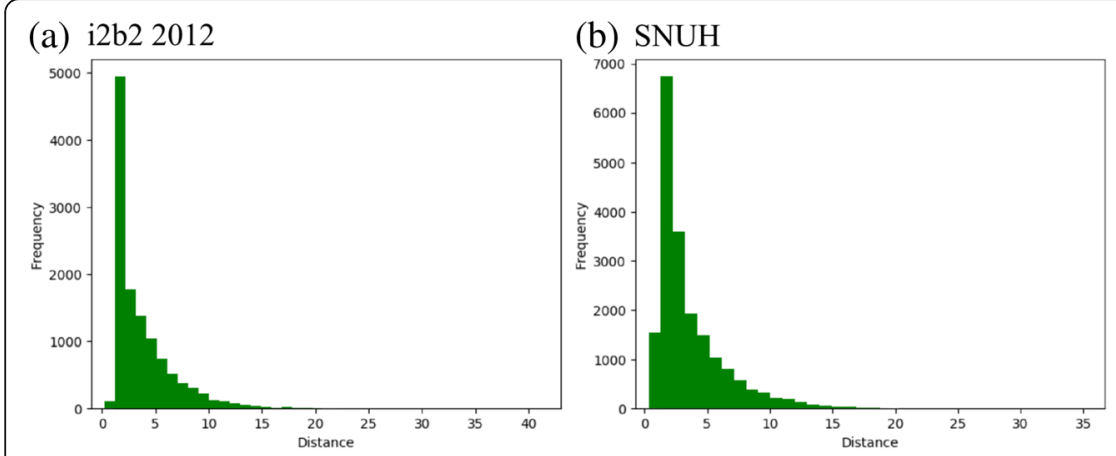

(c) CoNLL 2003

Fig. 5 Histograms of distances between named entities in each corpus. The number ' $n$ ' on the $x$-axis means $n$ non-entities exist within the two entities

capitalized or capitalization at the word beginning) [17]. Matching indicates whether a token matches a controlled vocabulary, e.g., the previous token is an obvious modifier of the current token, or a token is matched to a list consisting of the first entity tokens in the training data (frequency $>10$ ) as performed by $\mathrm{Li}$, et al. [43].

\section{Performance evaluation}

We used the three NER datasets to compare the proposed model structure with the first- and the second-order linear chain CRFs, and semi-Markov CRF [19], high-order CRF [18] that are variants of the CRF leveraging higher-order label transition dependency.

At first, we compared the pi-CRF with the first-order models. Table 5 shows the $F_{1}$ scores of the first-order CRF, the first-order CRF trained with the induced labels, and the pi-CRF for each test set. $F_{1}$ score is harmonic mean of the precision and recall scores. We first tested the models on all instances in each data set, and then tested the models on only those instances having two or more entities. The table shows that the proposed model structure offers a demonstrable improvement over the first-order models. The pi-CRF showed higher $\mathrm{F}_{1}$ scores for all feature settings on both the i2b2 2012 and the SNUH data sets.

In addition, the first-order CRF with induced labels shows the worst performance than others. Even though the induced label patterns can be easily obtained in the firstorder model, we can see that the use of the label induction without the 'observation symbol sharing' in the conventional model rather negatively affects its performance.

We also evaluated higher-order CRF models such as the conventional second-order CRF, semi-Markov CRF [19] and the high-order CRF [18, 20] implemented by A Allam and M Krauthammer [44]. The semi-Markov CRF and the high-order CRF are CRF variants using higherorder transition dependencies. The two CRF variants were trained with the stochastic gradient descent for 50 epochs. The results are reported in Table 6. As shown in the table, the pi-CRF shows a bit better performance than the other models in several settings and the pi-CRF also shows similar performance with the variants in a complex feature set.

In addition, we may observe the performance of the higher-order models including the pi-CRF were decreased in the general domain set (CoNLL 2003) in the simple feature settings. When we compare this result

Table 4 Summary of the feature settings. (The w denotes the window size. If the value is absent, only feature of the current token is used. The $n$ denotes the $n$ of the $n$-gram. The 'len' denotes the length of affixes. The matching features denote the result of controlled vocabulary matching)

\begin{tabular}{|c|c|c|c|c|c|c|c|}
\hline Set & Token & Norm-token & n-gram & character affix & capitalization & POS/Chunk & Matching \\
\hline$\# 1$-context & $w=3$ & $w=3$ & & & & & \\
\hline \#2-morph & $w=3$ & $w=3$ & & $\begin{array}{l}\text { len }=2 \sim 3 \\
w=3\end{array}$ & & & \\
\hline \#3-i2b2 & $w=5$ & $w=5$ & $\begin{array}{l}n=2 \\
w=5\end{array}$ & $\begin{array}{l}\text { len }=2 \sim 7 \\
W=3\end{array}$ & $w=1$ & & \\
\hline \#3-snuh & $w=5$ & $w=3$ & $\begin{array}{l}n=2 \\
w=5\end{array}$ & len $=2 \sim 3$ & & & modifier /control \\
\hline \#3-conll & $w=5$ & & & $\begin{array}{l}\text { len }=3 \sim 4 \\
w=5\end{array}$ & $w=5$ & $n=1$ & \\
\hline
\end{tabular}


Table $5 F_{1}$ scores of the first-order models and the pi-CRF for each corpora. The first value ('whole instance') is $F_{1}$ score with whole test set and the second value ('distanced instance') is $F_{1}$ score evaluated only with instances having transition dependency between NEs. (bold: best performance, shaded: pi-CRF)

\begin{tabular}{|c|c|c|c|c|c|c|c|}
\hline \multirow[t]{2}{*}{ Feature } & \multirow[t]{2}{*}{ Models } & \multicolumn{2}{|l|}{ i2b2 2012} & \multicolumn{2}{|l|}{ SNUH } & \multicolumn{2}{|c|}{ CoNLL 2003} \\
\hline & & $\begin{array}{l}\text { whole } \\
\text { instance }\end{array}$ & $\begin{array}{l}\text { distanced } \\
\text { instance }\end{array}$ & $\begin{array}{l}\text { whole } \\
\text { instance }\end{array}$ & $\begin{array}{l}\text { distanced } \\
\text { instance }\end{array}$ & $\begin{array}{l}\text { whole } \\
\text { instance }\end{array}$ & $\begin{array}{l}\text { distanced } \\
\text { instance }\end{array}$ \\
\hline \multirow[t]{3}{*}{ Set 1} & 1st-order CRF & 67.22 & 68.24 & 74.75 & 73.20 & 60.68 & 62.19 \\
\hline & $\begin{array}{l}\text { 1st-order CRF with induced } \\
\text { labels }\end{array}$ & 66.60 & 67.69 & 74.09 & 72.85 & 23.38 & 15.24 \\
\hline & pi-CRF & 67.29 & 68.43 & 75.50 & 74.43 & 45.54 & 43.41 \\
\hline \multirow[t]{3}{*}{ Set 2} & 1st-order CRF & 71.61 & 72.85 & 75.81 & 75.04 & 68.43 & 72.93 \\
\hline & $\begin{array}{l}\text { 1st-order CRF with induced } \\
\text { labels }\end{array}$ & 70.73 & 71.98 & 75.24 & 74.36 & 44.90 & 41.89 \\
\hline & $\mathrm{pi}-\mathrm{CRF}$ & 71.99 & 73.35 & 76.04 & 75.29 & 69.61 & 72.31 \\
\hline \multirow[t]{3}{*}{ Set 3} & 1st-order CRF & 72.55 & 73.97 & 76.18 & 75.06 & 82.57 & 83.13 \\
\hline & $\begin{array}{l}\text { 1st-order CRF with induced } \\
\text { labels }\end{array}$ & 71.25 & 72.75 & 75.37 & 74.18 & 80.81 & 81.55 \\
\hline & pi-CRF & 72.58 & 74.04 & 76.24 & 75.33 & 82.08 & 82.76 \\
\hline
\end{tabular}

with the corresponding tests in Table 5, the pi-CRF performs worse than the conventional models for the CoNLL data, though, we may interpret the performance decrease of the higher-order models in naïve feature setting might be expected.

Table 7 compares the proposed model's training and inference times using the feature setting \#3 with the conventional models. The table shows the numbers of parameters, states, elapsed training time, training time per iteration, and elapsed inference time. These values indicate that the pi-CRF design was slightly more complicated than the first-order CRF, although the proposed design was less complicated than the second-order CRF while still exploiting the transition information between NEs separated by long and arbitrary distances.

\section{Result analysis}

We also examined the model's behavior on the test data set. Table 8 shows the numbers of predicted entities and correct predictions on each held-out data set, using feature setting \#1. For the clinical data sets, the models that used longdistance transition dependency (i.e., the second-order and pi-CRF) tended to predict more entities than the first-order model, and the pi-CRF model correctly predicted more entities than both the first- and second-order CRF models, resulting in an improvement in recall performance: +0.7

Table $6 \mathrm{~F}_{1}$ scores of higher-order CRF models and pi-CRF for each corpora. The first value ('whole instance') is $F_{1}$ score with whole test set and the second value ('distanced instance') is $F_{1}$ score evaluated only with instanced having transition dependency between NEs. (bold: best performance, shaded: pi-CRF)

\begin{tabular}{|c|c|c|c|c|c|c|c|}
\hline \multirow[t]{2}{*}{ Feature } & \multirow[t]{2}{*}{ Models } & \multicolumn{2}{|l|}{ i2b2 2012} & \multicolumn{2}{|l|}{ SNUH } & \multicolumn{2}{|l|}{ CoNLL 2003} \\
\hline & & whole instance & distanced instance & whole instance & distanced instance & whole instance & distanced instance \\
\hline \multirow[t]{4}{*}{ Set 1} & 2nd-order CRF & 69.46 & 70.88 & 73.43 & 72.21 & 58.34 & 54.52 \\
\hline & semi-Markov CRF & 67.87 & 68.91 & 73.44 & 71.61 & 37.31 & 34.13 \\
\hline & high-order CRF & 68.38 & 69.52 & 73.50 & 71.69 & 36.97 & 33.87 \\
\hline & pi-CRF & 67.29 & 68.43 & 75.50 & 74.43 & 45.54 & 43.41 \\
\hline \multirow[t]{4}{*}{ Set 2} & 2nd-order CRF & 70.99 & 72.31 & 74.31 & 73.27 & 73.21 & 72.26 \\
\hline & semi-Markov CRF & 72.19 & 73.54 & 76.01 & 74.87 & 63.19 & 63.32 \\
\hline & high-order CRF & 71.50 & 72.74 & 76.11 & 74.97 & 63.56 & 63.76 \\
\hline & pi-CRF & 72.30 & 73.61 & 76.20 & 75.47 & 69.61 & 72.31 \\
\hline \multirow[t]{4}{*}{ Set 3} & 2nd-order CRF & 71.75 & 73.01 & 75.17 & 74.05 & 83.13 & 83.96 \\
\hline & semi-Markov CRF & 69.30 & 70.73 & 76.70 & 75.79 & 82.47 & 83.29 \\
\hline & high-order CRF & 69.26 & 70.64 & 76.73 & 75.91 & 82.18 & 82.80 \\
\hline & pi-CRF & 72.58 & 74.04 & 76.28 & 75.45 & 82.08 & 82.76 \\
\hline
\end{tabular}


Table 7 Efficiency test results. The numbers of parameters and states indicate the model's size. The elapsed training/inference times indicate the model's speed. (shaded: pi-CRF)

\begin{tabular}{lllllll}
\hline Data & Model & Parameter & State & Elapsed training time (sec) & Training time per iteration (sec) & $\begin{array}{c}\text { Elapsed } \\
\text { inference time (sec) }\end{array}$ \\
\hline i2b2 & 1st-order CRF & 442,705 & 8 & 1,550 & 12.5 & 1.7 \\
& 2nd-order CRF & 581,604 & 64 & 6,819 & 55.4 & 5.7 \\
& pi-CRF & 442,768 & 11 & 3,751 & 17.0 & 2.1 \\
SNUH & 1st-order CRF & 396,245 & 12 & 2,946 & 19.5 & 1.9 \\
& 2nd-order CRF & 495,772 & 144 & 27,388 & 139.7 & 9.3 \\
& pi-CRF & 396,400 & 17 & 6,231 & 23.6 & 2.1 \\
CoNLL & 1st-order CRF & 313,672 & 10 & 4,031 & 19.1 & 0.6 \\
& 2nd-order CRF & 431,044 & 100 & 24,828 & 173.6 & 2.6 \\
& pi-CRF & 313,776 & 14 & 13,512 & 29.4 & 0.7 \\
\hline
\end{tabular}

and +1.13 for the $\mathrm{i} 2 \mathrm{~b} 2$ and $\mathrm{SNUH}$, respectively. The final $\mathrm{F}_{1}$-score of the pi-CRF was improved than the first-order model, and we may indicate that the improvement of the recall consequently affects the improvement of the $F_{1}$-score of the pi-CRF. However, the models that used long-distance transition dependency (the second-order and the pi-CRF) showed the opposite behavior on the general data set, predicting noticeably fewer entities than the first-order model, although most of the higher-order models' predictions were correct. Thus, the precision performance of the pi-CRF showed an improvement of +16.4 for the CoNLL set, even though the recall performance was relatively low.

The models' expectation performance were additionally analyzed along the distances from the preceding entities. Trying to analyze the models according to the distance between the entities, we inevitably used the recall. Because this evaluation of the models with recall alone has its limitations, so this result was presented as an auxiliary indicator. The initial recall scores were calculated only for the entities not having precursors, and then the recall scores were updated sequentially by adding entities along the distances from 0 to the maximum distance for each data set. Figure 6 shows the analysis result. The graph of the models moved similarly along with the distance between entities: according to this figure, we can observe the recall scores of the CRF decrease as distance increases. The CRF models seem to miss the entities following when two entities are consecutive. We could not observe a significant performance improvement of the pi-CRF compared to other models. However, the pi-CRF shows better results in this result when this model was compared with the first-order CRF that uses a similar graphical structure with the pi-CRF. Especially, the performance of the first-order model, which was trained with induced labels, was remarkably decreased according to the distance. The use of the induced label is easy in the conventional model, but, it would not guarantee the performance improvement in the model without the observation symbol sharing. The models' recall scores have risen sharply at the points where distance is 1 in the i2b2 2012 and CoNLL. There is a small number of the entities having gap (order) value as 0 in

Table 8 The numbers of the models' expectation and the correct on each held-out set. (shaded: pi-CRF)

\begin{tabular}{|c|c|c|c|c|c|c|c|}
\hline \multirow[t]{2}{*}{ Data } & \multirow[t]{2}{*}{ Model } & \multicolumn{3}{|c|}{ Whole instances } & \multicolumn{3}{|c|}{ Distanced instances } \\
\hline & & gold & expected & correct & gold & expected & correct \\
\hline \multirow[t]{3}{*}{ i2b2 (clinical) } & 1st-order CRF & 9,623 & 7,361 & 5,708 & 8,552 & 6,188 & 4,927 \\
\hline & 2nd-order CRF & & 7,785 & 6,046 & & 6,547 & 5,245 \\
\hline & $\mathrm{pi}-\mathrm{CRF}$ & & 7,542 & 5,775 & & 6,397 & 5,012 \\
\hline \multirow[t]{3}{*}{ SNUH (clinical) } & 1st-order CRF & 17,125 & 15,326 & 12,128 & 12,520 & 10,813 & 8,540 \\
\hline & 2nd-order CRF & & 15,702 & 12,053 & & 11,088 & 8,524 \\
\hline & $\mathrm{pi}-\mathrm{CRF}$ & & 15,516 & 12,322 & & 11,012 & 8,758 \\
\hline \multirow[t]{3}{*}{ CoNLL (general) } & 1st-order CRF & 5,629 & 3,785 & 2,856 & 4,331 & 2,693 & 2,184 \\
\hline & 2nd-order CRF & & 2,778 & 2,529 & & 1,986 & 1,799 \\
\hline & pi-CRF & & 1,855 & 1,704 & & 1,280 & 1,218 \\
\hline
\end{tabular}




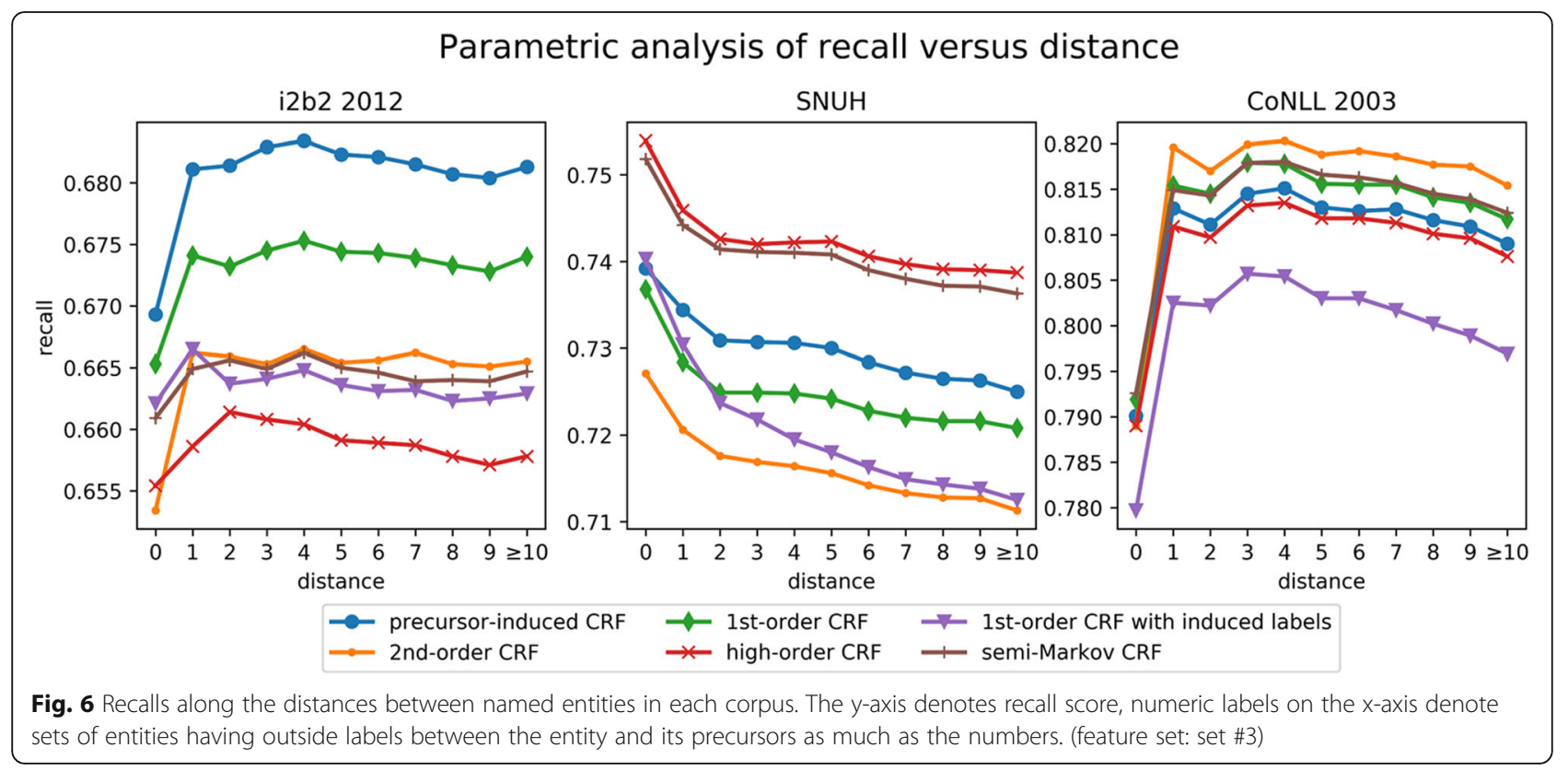

both data collections: the numbers of entities having gap value as zero are 50, 30, and 707 in the i2b2, CoNLL, and SNUH data respectively.

\section{Discussion}

In this study, we investigated the performance of the piCRF model which is a newly proposed variant of the CRF model designed particularly for extracting clinical NEs: the proposed model utilizes long-distance dependency relationships between the NEs separated by multiple non-entities in the CRF. The model fragments the non-entity state into fine-grained non-entity states and treats them as an information transmission medium based on the first-order linear chain CRF structure. The evaluation results showed that the proposed pi-CRF model is more effective at clinical NER. Although the piCRF model was slower than the first-order CRF, it was significantly faster than the second-order CRF model even while expressing higher-order transition dependencies between NEs.

Higher-order transitions are expressed as fixed-size label transitions in the conventional CRF model. Because the NEs tend to be separated by arbitrary distances, the conventional higher-order CRF model using a fixed-size state transition dependency has limited ability to express the desired information. One study of a semi-Markov CRF [19] proposed that consecutive units with the same label can be presented as a group although the model could not convey the information from the separated NEs. Based on this idea, we developed an induction method to present consecutive non-entity labels grouped by their precursor information. Besides, the mathematical formula (Eq. 3) used to express the proposed CRF model was derived from a CRF model that used virtual evidence [45], which incorporates prior knowledge of prototypes to make the model prefer to label consecutive values for a subsequence that matches a predefined pattern.

In contrast, our model used the formula to extend the hidden variables by joining two variables, $y$ and $a$. The two hidden variables are conjoined in Eq. 3: the variables are multiplied, and they are merged into a new hidden variable instead of using two hidden variables in the mathematics form. Because the variable $a$ has values only if the value of the corresponding $y$ is the non-entity state, the multiplication implies that the newly derived hidden variable $y^{\prime}$ has multiplied non-entity hidden states and the total number of the hidden states is expanded compared to the conventional CRF.

The design of the pi-CRF model improves the CRF model's expressive power according to the evaluation results. The transition information is implemented as feature functions, and thus the transition information ultimately affects the model as one of many features. Leveraging the high-order label transition information, the pi-CRF shows better performance than other higherorder CRF models in many evaluation settings. It could be the model's advantageous attribute that the proposed model preserves relatively compact model complexity than other higher-order models.

Avoiding the data sparseness problem was another significant concern in the model design. We expected the data sparseness problem to occur because the induction algorithm divides a single non-entity state into multiple states, and thus the frequency of observation features 
related to the outside label symbols was divided. In the model development phase, we observed that the model's performance was inferior without the feature sharing implemented by Eq. (4). For the clinical NER tasks, the results showed that the pi-CRF design increased the $F_{1}$ score compared with the first- and second-order CRF models while reducing the model's speed loss. Further improvement could be achieved by testing models trained with more sophisticated features on various data sets, or porting the model onto the state-of-the-art neural NER architecture with long short-term memory [15].

\section{Conclusion}

This study proposed a variant of the CRF model to improve the model's expressive power for clinical NER problems, in which NEs tend to be separated from nonentities. The proposed pi-CRF utilizes non-entity labels between NEs as an information transmission medium that delivers the preceding entity information forward to the following entity. Our evaluation results showed that the proposed model improves clinical NER performance and reduces the computational complexity of the second-order CRF. Despite some inherent limitations, the results suggest that the utilization of non-entity labels could enable higher-order CRF model implementation while limiting the model's complexity growth. We plan to test the model on various NER datasets and also to port the model onto a neural NER architecture [15] to further advance the clinical NER field.

\section{Abbreviations}

CoNLL: Conference on Natural Language Learning; CRF: Conditional Random Field; EHR: Electronic Health Record; i2b2: Information for Integrating Biology and the Bedsides; NE: Named Entity; NER: Named Entity Recognition; NLP: Natural Language Processing; pi-CRF: Precursor-Induced Conditional Random Fields; POS: Part-of-Speech; SNUH: Seoul National University Hospital

\section{Acknowledgments}

The de-identified English clinical documents were provided by the i2b2 project (US government project: U54LM008748) designed for the NLP Shared Tasks. The authors would like to thank Dr. Sungbin Choi, Jong Won Lim, and Ha Young Jo who actively participated in the generation of Korean clinical NER data.

\section{Authors' contributions}

Both authors contributed to the design of the study, analysis and interpretation of the data, drafting of the article, and final approval for submission. WL contributed to the conception of the study; carried out data collection, model implementation, and analysis; and drafted the manuscript, with significant contributions from JC. WL and JC performed data interpretation. Both authors read and approved the final manuscript.

\section{Funding}

This work was supported by the Basic Science Research Program through the National Research Foundation of Korea (NRF) funded by the Ministry of Education [No. NRF-2015R1D1A1A01058075]; and also supported by a grant of the Korea Health Technology R\&D Project through the Korea Health Industry Development Institute (KHIDI), funded by the Ministry of Health \&Welfare, Republic of Korea [grant number HI14C1277].

\section{Availability of data and materials}

The executable Java file is available at the GitHub repository https://github. com/jinsamdol/precursor-induced_CRF. However, all data were extracted from the medical record of patients who had been admitted at SNUH, so the clinical data cannot be shared with other research groups without permission.

\section{Ethics approval and consent to participate}

This study was approved by the Institutional Review Board (IRB) of Seoul National University Hospital (IRB No.1612-086-815). Informed consent was waived due to the retrospective nature of this study.

Consent for publication

Not applicable.

\section{Competing interests}

The authors declare that they have no competing interests.

\section{Author details}

${ }^{1}$ Interdisciplinary Program for Bioengineering, Graduate School, Seoul National University, 103 Daehak-ro, Jongno-gu, Seoul 03080, South Korea. ${ }^{2}$ Department of Biomedical Engineering, Seoul National University College of Medicine, 103 Daehak-ro, Jongno-gu, Seoul 03080, South Korea. ${ }^{3}$ Institute of Medical and Biological Engineering, Medical Research Center, Seoul National University, 101 Daehak-ro, Jongno-gu, Seoul 03080, South Korea.

Received: 24 August 2018 Accepted: 3 July 2019

Published online: 15 July 2019

\section{References}

1. Doan S, Conway M, Phuong TM, Ohno-Machado L. Natural language processing in biomedicine: a unified system architecture overview. Clin Bioinformatics, Methods Mol Biol. 2014;1168:275-94. https://doi.org/10.1007/ 978-1-4939-0847-9.

2. Patrick J, Li M. High accuracy information extraction of medication information from clinical notes: 2009 i2b2 medication extraction challenge. J Am Med Inform Assoc. 2010;17:524-7. https://doi.org/10.1136/jamia.2010.003939.

3. Sun W, Rumshisky A, Uzuner O. Evaluating temporal relations in clinical text: 2012 i2b2 challenge. J Am Med Inform Assoc. 2013:1-8. https://doi.org/1 0.1136/amiajnl-2013-001628.

4. Uzuner Ö, South BR, Shen S, DuVall SL. 2010 i2b2NA challenge on concepts, assertions, and relations in clinical text. J Am Med Inform Assoc. 2011;18:552-6. https://doi.org/10.1136/amiajnl-2011-000203.

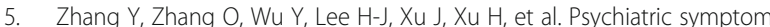
recognition without labeled data using distributional representations of phrases and on-line knowledge. J Biomed Inform. 2017;75S:S129-37.

6. Jiang M, Chen Y, Liu M, Rosenbloom ST, Mani S, Denny JC, et al. A study of machine-learning-based approaches to extract clinical entities and their assertions from discharge summaries. J Am Med Inform Assoc. 2011;18:6016. https://doi.org/10.1136/amiajnl-2011-000163.

7. Murff HJ, FitzHenry F, Matheny ME, Gentry N, Kotter KL, Crimin K, et al. Automated identification of postoperative complications within an electronic medical record using natural language processing. JAMA - J Am Med Assoc. 2011;306:848-55. https://doi.org/10.1001/jama.2011.1204.

8. Nadkarni PM, Ohno-Machado L, Chapman WW. Natural language processing: an introduction. J Am Med Inform Assoc. 2011;18:544-51. https://doi.org/10.1136/amiajnl-2011-000464.

9. Yeh A, Morgan A, Colosimo M, Hirschman L. BioCreAtlvE task 1A: gene mention finding evaluation. BMC Bioinformatics. 2005;6(Suppl 1):S2. https:// doi.org/10.1186/1471-2105-6-S1-S2.

10. Lafferty J, McCallum A, Pereira F. Conditional random Fields : probabilistic models for segmenting and labeling sequence data. In: Proceedings of the 18th international conference on machine learning, vol. 2001; 2001. p. 282-9.

11. McCallum A, Li W. Early results for named entity recognition with conditional random fields, feature induction and web-enhanced lexicons. In: Proceeding of CoNLL, vol. 2003; 2003. p. 188-91.

12. Sha F, Pereira F. Shallow parsing with conditional random fields. In: Proceedings of the 2003 conference of the north American chapter of the Association for Computational Linguistics on human language technology; 2003. p. 134-41. 
13. McDonald R, Pereira F. Identifying gene and protein mentions in text using conditional random fields. BMC Bioinformatics. 2005;6(Suppl 1):S6. https:// doi.org/10.1186/1471-2105-6-S1-S6.

14. Bethard S, Savova G, Chen W-T, Derczynski L, Pustejovsky J, Verhagen M. SemEval-2016 Task 12: Clinical TempEval. Proc 10th Int Conf Semant Eva (SemEval 2016); 2016. p. 1052-62. https://doi.org/10.18653/v1/S16-1165.

15. Lample G, Ballesteros M, Subramanian S, Kawakami K, Dyer C. Neural architectures for named entity recognition. In: Proceedings of NAACL-HLT 2016; 2016. p. 260-70

16. Liu Z, Yang M, Wang X, Chen Q, Tang B, Wang Z, et al. Entity recognition from clinical texts via recurrent neural network. BMC Med Inform Decis Mak. 2017;17(Suppl 2):53-60

17. Ratinov L, Roth D. Design challenges and misconceptions in named entity recognition. In: Proceedings of the thirteenth conference on computational natural language learning; 2009. p. 147-55.

18. Ye N, Lee WS, Chieu HL, Wu D. Conditional random fields with high-order features for sequence labeling. In: Advances in neural information processing systems; 2009. p. 2196-204.

19. Sarawagi S, Cohen WW. Semi-Markov conditional random fields for information extraction. In: Advances in neural information processing systems; 2005. p. 1185-92.

20. Cuong NV, Ye N, Lee WS, Chieu HL. Conditional random field with highorder dependencies for sequence labeling and segmentation. ACM JMLR. 2014;15:981-1009

21. Fersini $E$, Messina E, Felici G, Roth D. Soft-constrained inference for named entity recognition. Inf Process Manag. 2014;50:807-19. https://doi.org/10.1 016/j.ipm.2014.04.005.

22. Li $X$, Wang $Y-Y$, Acero $A$. Extracting structured information from user queries with semi-supervised conditional random fields. In: Proc 32nd Int ACM SIGIR Conf res dev Inf Retr - SIGIR '09; 2009. p. 572. https://doi.org/10.1145/1571 941.1572039.

23. Li L, Jin L, Jiang Z, Song D, Huang D. Biomedical named entity recognition based on extended Recurrent Neural Networks. In: Proc - 2015 IEEE Int Conf Bioinforma biomed BIBM 2015; 2015. p. 649-52.

24. Chalapathy R, Borzeshi EZ, Piccardi M. Bidirectional LSTM-CRF for clinical concept extraction. In: Proceedings of the clinical natural language processing workshop; 2016. p. 7-12. http://arxiv.org/abs/1611.08373.

25. Dernoncourt F, Lee JY, Uzuner O, Szolovits P. De-identification of patient notes with recurrent neural networks. J Am Med Informatics Assoc. 2017;24:596-606.

26. Jauregi Unanue I, Zare Borzeshi E, Piccardi M, et al. J Biomed Inform. 2017; 76:102-9. https://doi.org/10.1016/j.jbi.2017.11.007.

27. Jagannatha $\mathrm{A}, \mathrm{Yu} \mathrm{H}$. Bidirectional recurrent neural networks for medical event detection in electronic health records. In: NAACL-HLT; 2016. p. 47382. http://arxiv.org/abs/1606.07953.

28. Sahu SK, Anand A. Recurrent neural network models for disease name recognition using domain invariant features. In: Proceedings of the 54th annual meeting of the Association for Computational Linguistics; 2016. p. 2216-25. http://arxiv.org/abs/1606.09371.

29. Kholghi M, Sitbon L, Zuccon G, Nguyen A. Active learning: a step towards automating medical concept extraction. J Am Med Informatics Assoc. 2016;23: 289-96.

30. Hao T, Pan X, Gu Z, Qu Y, Weng H. A pattern learning-based method for temporal expression extraction and normalization from multi-lingual heterogeneous clinical texts. BMC Med Inform Decis Mak. 2018;18(Suppl 1):22.

31. Wang P, Hao T, Yan J, Jin L. Large-scale extraction of drug-disease pairs from the medical literature. J Assoc Inf Sci Technol. 2017;68:2649-61.

32. Stubbs A, Kotfila C, Xu H, Uzuner Ö. Identifying risk factors for heart disease over time: overview of 2014 i2b2/UTHealth shared task track 2. J Biomed Inform. 2015;58:567-77.

33. Soysal E, Wang J, Jiang M, Wu Y, Pakhomov S, Liu H, et al. CLAMP - a toolkit for efficiently building customized clinical natural language processing pipelines. J Am Med Informatics Assoc. 2018;25:331-6.

34. Demner-Fushman D, Rogers WJ, Aronson AR. MetaMap lite: an evaluation of a new Java implementation of MetaMap. J Am Med Informatics Assoc. 2017;24:841-4

35. Sutton C, McCallum A. An introduction to conditional random fields. Found Trends Mach Learn. 2011:4:267-373.

36. Tjong EF, Sang K. Representing text chunks; 1995. p. 173-9.

37. Freitag D, McCallum A. Information extraction with HMM structures learned by stochastic optimization. In: AAAl; 2000.
38. Andrew Kachites MCCallum. MALLET: a machine learning for language toolkit. 2002. http://mallet.cs.umass.edu. Accessed 27 Mar 2013.

39. $\mathrm{Ng}$ AY. Feature selection, L1 vs. L2 regularization, and rotational invariance. In: ICML 2004; 2004

40. Lee W, Kim K, Lee EY, Choi J. Conditional random fields for clinical named entity recognition: a comparative study using Korean clinical texts. Comput Biol Med. 2018;101:7-14.

41. Tjong EF, Sang K, De MF. Introduction to the CoNLL-2003 shared Task : languageindependent named entity recognition. In: Proceedings of the seventh conference on natural language learning at HLT-NAACL 2003; 2003. p. 142-7.

42. Xu Y, Wang Y, Liu T, Tsujii J, El-C C. An end-to-end system to identify temporal relation in discharge summaries: 2012 i2b2 challenge. J Am Med Inform Assoc. 2013;20:849-58. https://doi.org/10.1136/amiajnl-2012-001607.

43. Li L, Zhou R, Huang D. Two-phase biomedical named entity recognition using CRFs. Comput Biol Chem. 2009:33:334-8.

44. Allam A, Krauthammer M. PySeqLab an open source Python package for sequence labeling and segmentation. https://pyseglab.readthedocs.io.

45. Li X. On the Use of Virtual Evidence in Conditional Random Fields; 2009. p. 1289-97

\section{Publisher's Note}

Springer Nature remains neutral with regard to jurisdictional claims in published maps and institutional affiliations.
Ready to submit your research? Choose BMC and benefit from:

- fast, convenient online submission

- thorough peer review by experienced researchers in your field

- rapid publication on acceptance

- support for research data, including large and complex data types

- gold Open Access which fosters wider collaboration and increased citations

- maximum visibility for your research: over $100 \mathrm{M}$ website views per year

At $\mathrm{BMC}$, research is always in progress.

Learn more biomedcentral.com/submissions 